\title{
Facile analytical extraction of the hyperelastic constants for the two-parameter Mooney-Rivlin model from experiments on soft polymers
}

\author{
Tilvawala Gopesh and James Friend
}

June 30, 2021

\begin{abstract}
Having accurate data to represent hyperelastic materials that underpin soft robotics would facilitate their analysis, design, and validation. We seek to provide the reader with a useful tool to overcome a mundane but crucially important problem in determining the hyperelastic material properties. We show how to employ first dimensionless and then dimensional comparisons between experimental data and the classic theoretical model representing this system to produce $C_{1}$ and $C_{2}$ for the Mooney-Rivlin model, closely representing a variety of soft polymers.
\end{abstract}




\section{Introduction}

Soft robotics has increasingly gained traction in research and applications over the last decade [1]. This typically involves making actuators of different sizes and configurations using hyperelastic materials, biological materials, or synthetic soft tissues [2] to perform dextrous maneuvers that would otherwise be impossible to conduct using conventional rigid robots. Hyperelastic materials can be manufactured with biocompatibility and a broad range of elastic moduli, failure strains from $200 \%$ to over $1000 \%$, surface properties, solvent resistance, and thermal resistance. The material choice depends upon the target application, making it one of the most critical parameters in research design and development. The exponential growth of the soft robotics discipline has been driving a similar growth in the number and variety of hyperelastic materials. Understanding and controlling their behavior in applications is crucial to continue this growth.

In order to design and characterize the behavior of hyperelastic polymer materials, various constitutive models have been developed in the past. Beyond the classic Neo-Hookean model [3], the theory for large deformations of hyperelastic polymers was initiated by the seminal work of Mooney [4] and further elaborated by Rivlin and Saunders [5] which has come to define the Mooney-Rivlin model. Other well-known models include the Ogden [6], Yeoh [7], Blatz-Ko [8], Arruda-Boyce [9], and Gent [10] models. Each of these constitutive models require one or more hyperelastic constants, and finding values for those constants typically require a regimen of experiments.

In the case of the Neo-Hookean model, uniaxial tests are sufficient to fully determine the single constant that it requires to define the material. However, most other models require more than one material constant, and so more experimental tests are required, usually among the following choices: pure shear, equibiaxial tension, planar tension, uniaxial compression, uniaxial tension, and biaxial membrane (bulge) tests [11]. An alternative approach using atomic force microscopy nanoindentation tests was studied by Dimitriadis, et al. [12], and subsequently in a few more studies [13, 14, 15] where parameters $B_{1}$ and $B_{2}$ represent the hyperelastic constants. This approach requires extraction of $B_{1}$ and $B_{2}$ using the Young's modulus $E$, however there is no established relationship between $B_{1}, B_{2}, C_{1}$, and $C_{2}$. There has been demonstration of a purely computational approach, without experimental validation, that involves a finite element model (using ABAQUS, Dassault 
Systèmes, Vélizy-Villacoublay, France) comparing the results obtained from neo-Hookean, Mooney-Rivlin and Yeoh models. Other approaches involve extraction of the storage modulus [13, 16] to iteratively tailor the behavior of PDMS. The tensile and compressive moduli can also be extracted using [17] tensile and compressive testing. In the two-parameter Mooney-Rivlin model, second-order Yeoh and second-order Ogden models, two hyperelastic constants are required. For instance, the first and second hyperelastic constants $C_{1}$ and $C_{2}$, also referred to as $C_{10}$ and $C_{01}$, respectively, in the two-parameter Mooney-Rivlin case. While uniaxial tensile tests are sufficient to obtain the first hyperelastic constant $C_{1}$, biaxial membrane tests are typically necessary to obtain the second hyperelastic constant $C_{2}$. Various methods and results have been presented to extract the hyperelastic constants [18, 19], however, there are limited benchmark results for the values of hyperelastic constants of common materials such as polydimethylsiloxane (PDMS, Sylgard ${ }^{\mathrm{TM}}$ 184, Dow Corning Corp., Midland, MI USA) and many other proprietary hyperelastic materials, such as the platinum-cure silicone Dragon Skin (Smooth-On Corp., Macungie, PA USA).

The first reported values for PDMS were $C_{1}=34.3 \mathrm{kPa}$ and $C_{2}=46.9 \mathrm{kPa}[20]$, obtained from uniaxial tensile tests. In a subsequent study, uniaxial experiments were conducted in both axisymmetric and asymmetric fashion, and computational simulations using constitutive models were used to compare against experimental data and establish material constant values for the models [21]. Sasso et al. [11] added biaxial tests to uniaxial tests on rubber-like materials and validated the results with finite element analysis (ABAQUS) to determine the values of the material constants for the Neo-Hookean, Arruda-Boyce, Mooney-Rivlin, Ogden, and Yeoh material models. Later, the results of ultra-large deformation bulge tests conducted on PDMS microballoons were compared to finite element analysis (ANSYS, Inc., Canonsburg, PA USA) to obtain $C_{1}=75.5 \mathrm{kPa}$ and $C_{2}=5.7 \mathrm{kPa}$ for PDMS [22], remarkably different than Kawamura et al. [20]. Uniaxial tensile and compression tests of dielectric elastomers have been used in conjunction with finite element analysis (ABAQUS) to determine the properties of dielectric elastomers [23], though these materials would certainly be expected to exhibit mechanical properties different than pure PDMS.

The overall approach in the literature has been to validate experimentally-obtained results with finite element analysis, and then use the results of this validation to derive the appropriate constants necessary to represent the material with an analytical model. While the use of computation to 
compare with experimental results and determine the constants for use in an analytically-derived model is convenient, it is tedious and subject to modeling and representation errors made in finite element analysis. Since it is actually possible to directly use the theory in determining the appropriate values for representative constants such as $C_{1}$ and $C_{2}$-without having to resort to computational analysis-a viable alternative is to omit the computations. This avoids potential problems arising from approximate representation of the boundary conditions and interfaces present in the system when using finite element analysis. The theoretical approach likewise eliminates the computational costs associated with obtaining and running FEA models.

We consider as an example of this approach the inflation of a small cylindrical disk of hyperelastic media restrained at the periphery. Christensen and Feng [24] long ago combined the theory developed by Mooney [4] and Rivlin [25] with an approximate closed-form solution for the inflation of a thin circular disk to determine the hyperelastic material constants, but there has been no demonstration since to use this theory to produce, for example, the $C_{1}$ and $C_{2}$ values for PDMS. In other words, no one has used a purely analytical approach to validate the experimental data. However, over this same time many [21, 11, 26] have used the comparatively painstaking approach of introducing computations to determine these values, as they enable analogous representations of the respective experiments conducted on the hyperelastic materials. In this paper, a non-dimensionalized analytical validation method is provided as a universally applicable approach regardless of the details, provided the minimum criterion for the stretch ratio is met as discussed later.

\section{Method}

\subsection{Experiments}

Different hyperelastic materials will exhibit independent hyperelastic constants. Uniaxial tensile tests are frequently employed to extract the first hyperelastic constant $C_{1}$, however to obtain the both $C_{1}$ and $C_{2}$, a biaxial membrane test is required. Biaxial tests can be conducted in different configurations, either beginning with a three-dimensional balloon or biaxially inflating an initially flat disk. Making uniform flat, thin films is easier than fabricating three-dimensional balloons with good surface uniformity out of unknown materials, and so we adopt the biaxially inflated flat disk approach. 
Thin films of five different hyperelastic materials, PDMS (Sylgard $\left.{ }^{\mathrm{TM}} 184\right)$, Dragon-Skin ${ }^{\mathrm{TM}} 10$, Eco-flex ${ }^{\mathrm{TM}} 30$, Sorta-clear ${ }^{\mathrm{TM}} 40$, and Dragon-Skin ${ }^{\mathrm{TM}}$ mixed with hexane (weight ratio Dragon-Skin A:B:hexane $=1: 1: 1$, were made made by combining the base and curing agent in the manufacturer recommended ratio. Glass slides were coated with release agent (Ease Release ${ }^{\mathrm{TM}} 200$, Mann Release Technologies, Macungie, PA, USA). All the hyper-elastic polymers were spin-coated at 750 RPM for 60 seconds. Upon curing, the resulting thin films were placed in a custom fixture shown in Fig. 1.

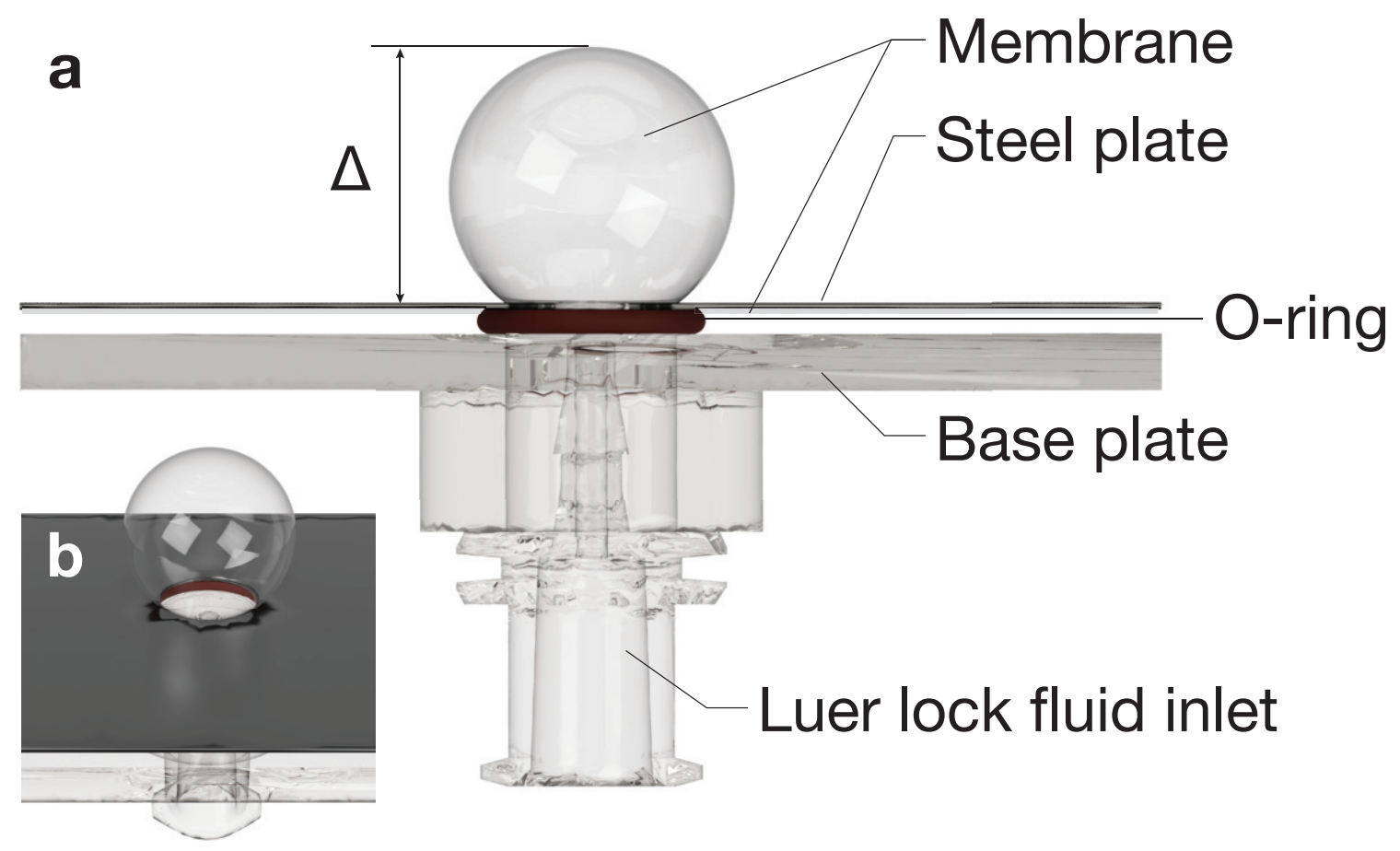

Figure 1: a) A Luer connector base and steel plate with hole provided to pass the membrane through when inflated, shown (b) from slightly above the horizontal view. The membrane, upon hydraulic inflation from the Luer lock expands as a nearly spherical shape to produce a vertical height above the steel plate, $\Delta$, that grows with inflation until membrane failure. The O-ring beneath clamps the membrane in place against the steel plate.

The 3-mm diameter of the circular hole in the base plate and steel plate are similar to eliminate potential lateral stretching. The $4-\mathrm{mm}$ diameter, $0.5-\mathrm{mm}$ thickness O-ring prevents air leakage 
between the Luer connector base and the steel plate; it is sized to be commensurate with the inner diameter of the steel plate to prevent interference with the membrane expansion from the circular hole. The entire assembly ensures the entire membrane's edge along a circular section is clamped together, leaving a $3 \mathrm{~mm}$ radius circular region free to inflate in the middle from an inlet in the substrate to which the membrane and clamp were fastened. Water was introduced through a Luer lock connection into the chamber formed by the clamped membrane and inlet using a high precision microfluidic system (microfluidics control, OB1, Elveflow ${ }^{\circledR}$, Paris, France). Water was used as the driving fluid to eliminate the potential effects of compressibility associated with pneumatic (air or gas) inflation. The membranes were inflated to failure with pressure increments of $3 \mathrm{kPa} /$ second and filmed using a high-speed camera (Fastcam Mini UX100, Photron, Irvine, CA, USA) combined with a long-distance microscope (CF-1, Infinity, Boulder, CO, USA). The Elveflow system provided the inflation pressure as a function of time to a computer, and was used to trigger the camera to synchronize inflation of the membrane with video recording saved on the same computer. The deformation of the membranes as a function of the inflation pressure was obtained using custom image processing code on MATLAB (MATLAB, Natick, MA, USA).

\subsection{Analysis}

In order to evaluate the hyperelastic constant values, a relation between the inflation pressure and deformation of the membrane must be established. For a hyperelastic material, the Mooney-Rivlin form of the strain energy function, $W$ is defined as [4, 5]

$$
W=C_{1}\left(J_{1}-3\right)+C_{2}\left(J_{2}-3\right)
$$

The constant $C_{1}$ is proportional to the number of molecular strands per unit volume, and $C_{2}$ represents additional restraints on the molecular strands; the shear modulus $G=2\left(C_{1}+C_{2}\right)$ while $J_{1}$ is a measure of the strain defined as [27]

$$
J_{1}=\lambda_{1}^{2}+\lambda_{2}^{2}+\lambda_{3}^{2}
$$


and $J_{2}$ is defined as

$$
J_{2}=\lambda_{1}^{-2}+\lambda_{2}^{-2}+\lambda_{3}^{-2}
$$

When an initially flat, circular disc of an elastomeric material with radius $R$ is inflated in a biaxial membrane, or "bulge", test to produce a spherical cap shape, the stretch ratio, $\lambda$, for the maximum displacement of the membrane, $\Delta$, is defined as [24]

$$
\lambda=\left(\frac{\frac{\Delta}{R}+\frac{R}{\Delta}}{2}\right) \sin ^{-1}\left(\frac{2}{\frac{\Delta}{R}+\frac{R}{\Delta}}\right) .
$$

The relationship between the inflating pressure, $P$, the maximum deformation of the membrane, $\Delta$, and the stretch ratio, $\lambda$ is defined as [24]

$$
P=\frac{8 C_{1} H}{R\left(\frac{\Delta}{R}+\frac{R}{\Delta}\right)}\left[\left(1-\frac{1}{\lambda^{6}}\right)+\alpha\left(\lambda^{2}-\frac{1}{\lambda^{4}}\right)\right],
$$

where $H$ is the thickness of the membrane and $\alpha=C_{2} / C_{1}$ is the ratio of the hyperelastic constants. Non-dimensionalizing the pressure and the deformation as

$$
\tilde{P}=\frac{R P}{4 C_{1} H} \quad \text { and } \quad \tilde{\Delta}=\frac{\Delta}{R_{0}},
$$

where $R_{0}$ is the initial radius of the membrane, produces

$$
\tilde{P}=\frac{2}{\left(\tilde{\Delta}+\frac{1}{\tilde{\Delta}}\right)}\left[\left(1-\frac{1}{\lambda^{6}}\right)+\alpha\left(\lambda^{2}-\frac{1}{\lambda^{4}}\right)\right],
$$

where

$$
\lambda=\left\{\begin{array}{ccc}
\frac{\left(\tilde{\Delta}+\frac{1}{\tilde{\Delta}}\right)}{2} \sin ^{-1}\left(\frac{2}{\frac{\tilde{\Delta}}{R}+\frac{R}{\tilde{\Delta}}}\right) & \text { if } & \tilde{\Delta} \leq 1 \\
\frac{\left(\tilde{\Delta}+\frac{1}{\tilde{\Delta}}\right)}{2}\left(\pi-\sin ^{-1}\left(\frac{2}{\frac{\tilde{\Delta}}{R}+\frac{R}{\tilde{\Delta}}}\right)\right) & \text { if } & \tilde{\Delta} \geq 1
\end{array} .\right.
$$

The dimensionless analytical pressure $\tilde{P}$ was obtained as a function of the stretch ratio $\tilde{\Delta}$ using eqn. [6. The experimental $P_{\exp }$ was scaled by $\left.P_{R} \equiv P_{\exp }\right|_{\Delta=R}$ to produce the nondimensional pressure $\tilde{P}_{\exp }=P_{\exp } / P_{R}$. The pressure $P_{R}$ is the pressure required for the membrane to deform an amount equivalent to the initial membrane radius, $\Delta=R$. For an increase $d r$ of the membrane radius, the 
expansion of the surface requires an amount of work proportional to the pressure. The hyperelastic constants define the factor of proportionality. Least-squares minimization was used to determine the best value of $\alpha$ in minimizing the error between the dimensionless experimental pressure-deformation data and the analytical dimensionless pressure obtained from eqn. (6) across the stretch ratios. Since the materials tested in this study have different mechanical properties, the values of $P_{R}$ and $\alpha$ are different for each material.

After establishing the value of $\alpha$, the dimensional pressure $P$ was evaluated in terms of the dimensional deformation $\Delta$ using eqn. (5), where the thickness of the membranes was obtained using a surface profilometer (Dektak 150, Veeco, Plainview, NY, USA). Least-squares minimization was performed to determine the value of $C_{1}$ that minimizes the error between the dimensional experimental pressure-deformation data and the analytical pressure-deformation obtained from eqn. (1). Since the value of $\alpha$ is known from the previous step, $C_{2}=C_{1} \alpha$ can also be determined.

\section{Results}

The experimental nondimensionalized pressure is plotted with respect to the stretch for two of the materials, Ecoflex ${ }^{\mathrm{TM}}$ and Sylgard ${ }^{\mathrm{TM}} 184$ (PDMS), as shown in Fig. 1. The remaining materials' plots are provided in the supplementary information. 
a)

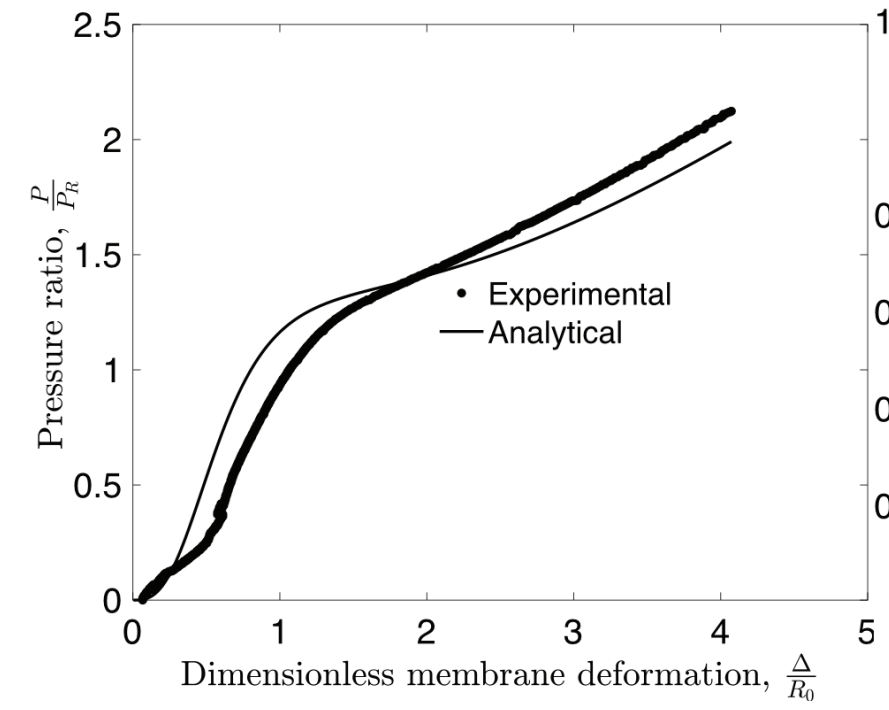

b)

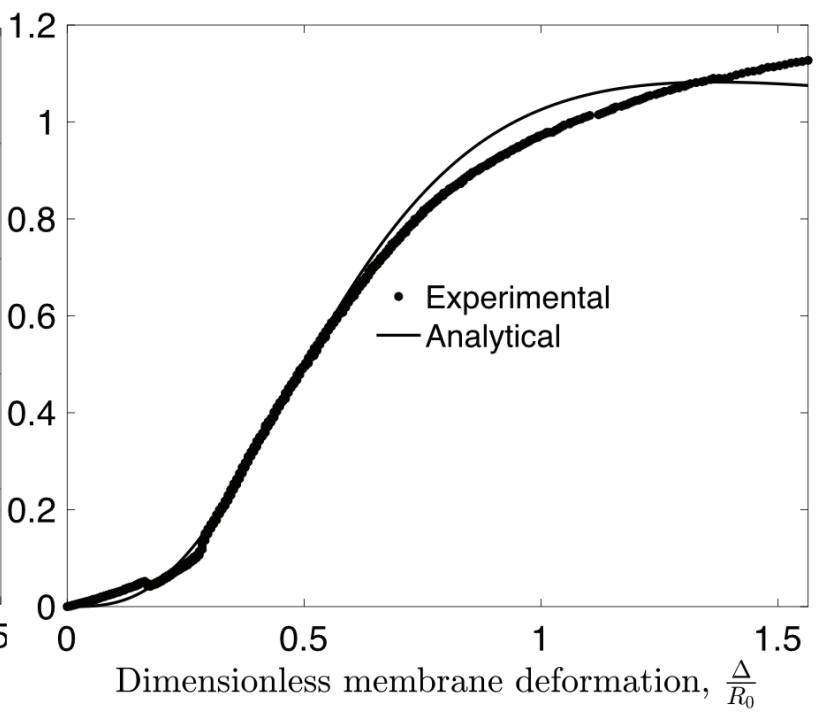

Figure 2: Dimensionless inflation pressure, $P / P_{R}$ plotted with respect to the dimensionless deformation of the membrane, $\Delta / R_{0}$ (x-axis) for a) Ecoflex ${ }^{\mathrm{TM}}$ and b) Sylgard ${ }^{\mathrm{TM}} 184$ (PDMS). Least-squares minimization was used to determine the value of the ratio of the hyperelastic constants, $\alpha$, to directly fit the non-dimensionalized analytical model to the non-dimensionalized experimental data (Ecoflex: $\alpha=0.1, R^{2}=0.91$; PDMS: $\alpha=0.04, R^{2}=0.99$ ).

Least squares minimization was used between the experimental data and eqn. (3) to determine the value of $\alpha$ and obtain the best-fit plots in Fig.1.

Once the value of ratio of the hyperelastic constants, $\alpha$, is found, the value of $C_{1}$ may then be found by least squares fitting of eqn. (1) to the dimensional pressure-deformation results as illustrated in Fig. 3 . 
a)

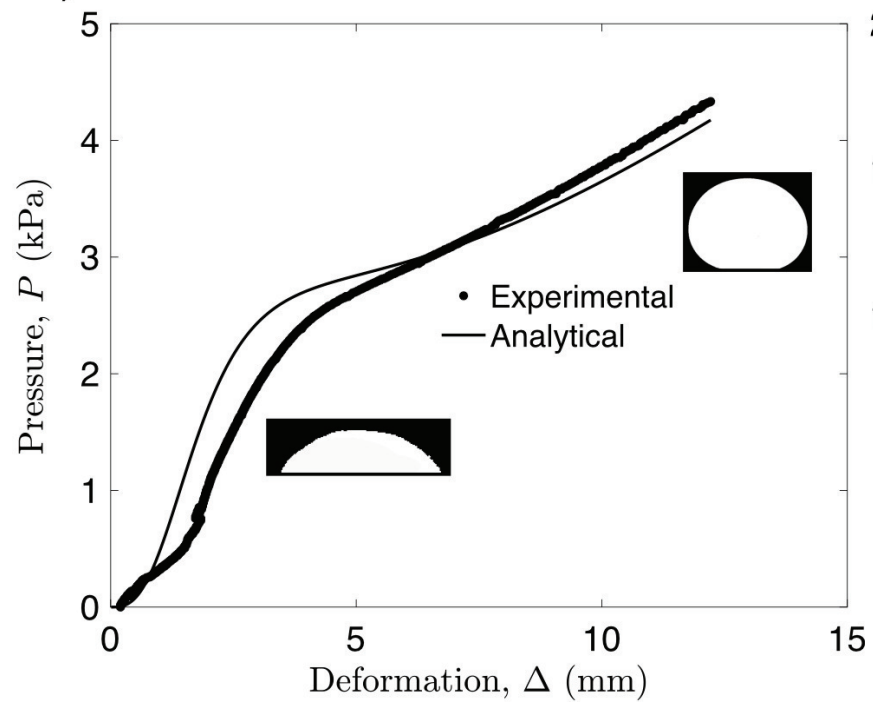

b)

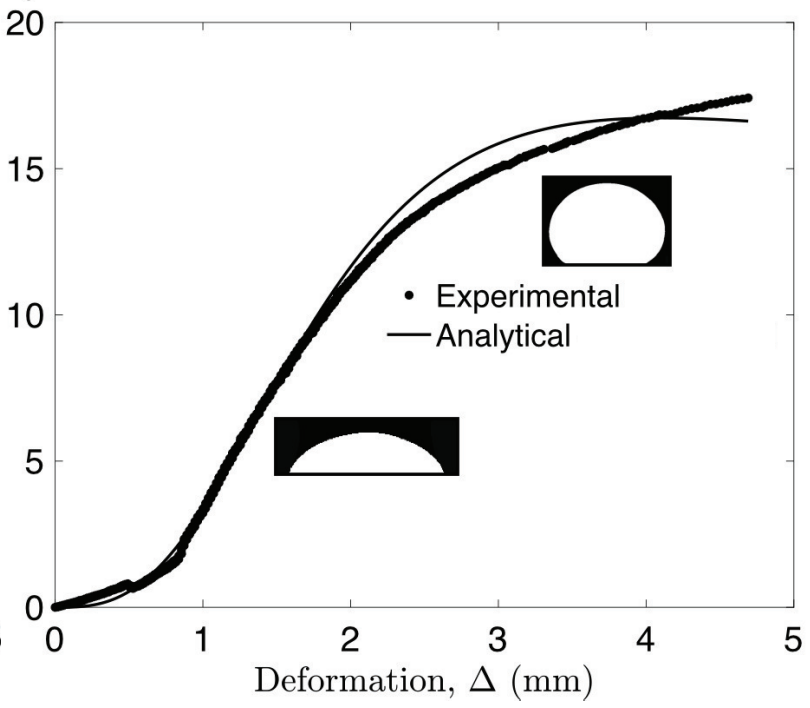

Figure 3: Dimensional inflation pressure, $P$ as a function of the maximum deformation of the membrane, $\Delta$ for a) Ecoflex ${ }^{\mathrm{TM}}$ and b) Sylgard ${ }^{\mathrm{TM}} 184$ (PDMS). Least squares minimization was used to find the best fit for $C_{1}$ knowing the appropriate value of $\alpha$ from the dimensionless fitting. The values of $C_{1}$ found from this procedure are provided in Table 1 and $R^{2}=0.89$ (Ecoflex) and $R^{2}=0.99$ (PDMS).

Table1 1 lists all the materials tested in this study, and their respective values of $C_{1}$ and $C_{2}$, indicating an ability to test a rather broad range of materials, and likewise notably indicating the remarkable range possible from relatively similar soft polymer materials.

Table 1: Values of hyperelastic constants $C_{1}$ and $C_{2}=\alpha C_{1}$ obtained by fitting the two-parameter Mooney-Rivlin model.

\begin{tabular}{|c|c|c|}
\hline Material & $C_{1}(\mathrm{kPa})$ & $C_{2}(\mathrm{kPa})$ \\
\hline Dragon-Skin ${ }^{\mathrm{TM}} 10$ & 180 & 11.7 \\
\hline Ecoflex ${ }^{\mathrm{TM}}$ & 75 & 7.5 \\
\hline Sorta-Clear ${ }^{\mathrm{TM}} 40$ & 830 & 52.3 \\
\hline Sylgard $^{\mathrm{TM}} 184$ (PDMS) & 270 & 10.8 \\
\hline Dragon-Skin ${ }^{\mathrm{TM}} 10+$ Hexane at $1: 1$ weight ratio & 180 & 14.9 \\
\hline
\end{tabular}

Table 2 shows the values of the hyperelastic constants $C_{1}$ and $C_{2}$ obtained for PDMS compared to previously published results. 
Table 2: Comparison of hyperelastic constant values for Sylgard ${ }^{\mathrm{TM}} 184$ (PDMS) through the analytical approach and previous studies with biaxial membrane (bulge) tests.

\begin{tabular}{lcr}
\hline Sylgard $^{\mathrm{TM}}$ 184 (PDMS) & $C_{1}(\mathrm{kPa})$ & $C_{2}(\mathrm{kPa})$ \\
\hline Analytical approach & 270.0 & 10.8 \\
Kawamura et al. [20] & 34.3 & 46.9 \\
Yoon et al. [22] & 75.5 & 5.7 \\
\hline
\end{tabular}

\section{Conclusions}

Biaxial membrane tests were conducted on five different hyperelastic materials. High resolution imagery, high precision microfluidic systems, and custom MATLAB image processing code were used to extract the inflation pressure as a function of the stretch ratio for the hyperelastic membranes. Each of the membranes were tested to failure to ensure the minimum criterion for the stretch ratio was met. As an example, the two-parameter Mooney-Rivlin model was used in conjunction with an analytical approximation for the large deformation of elastic discs to establish the hyperelastic material properties of five different hyperelastic materials. This approach, although quite simplified, is applicable to a large variety of hyperelastic materials enabling the extraction of their hyperelastic constants through analytical validation of the experimental data. Although the values of the hyperelastic constants in previous studies substantially differ between themselves and the values we found, this can be attributed to different material formulations [16] and curing conditions, particularly temperature and cure duration [17].

However, the facile method presented in this paper provides a means to easily determine the mechanical characteristics of a hyperelastic material. The method avoids computational analysis which introduces problems of its own, and can be quickly performed so that many material choices can be considered in design. Because it is simple to set up and conduct, the method also enables the reader to quickly produce useful property values from their own materials that may be then used in more accurately modeling their robots and other devices. 


\section{References}

[1] George M Whitesides. Soft robotics. Angewandte Chemie International Edition, 57(16):4258-4273, 2018.

[2] Larry Alan Taber. Nonlinear theory of elasticity: applications in biomechanics. World Scientific, 2004.

[3] Robert Hooke. 1678. De Potentia restitutiva, 1931.

[4] Melvin Mooney. A theory of large elastic deformation. Journal of applied physics, 11(9):582-592, 1940.

[5] Ronald S Rivlin and DW Saunders. Large elastic deformations of isotropic materials vii. experiments on the deformation of rubber. Philosophical Transactions of the Royal Society of London. Series A, Mathematical and Physical Sciences, 243(865):251-288, 1951.

[6] Raymond W Ogden. Non-linear elastic deformations. Courier Corporation, 1997.

[7] Oon H Yeoh. Some forms of the strain energy function for rubber. Rubber Chemistry and technology, 66(5):754-771, 1993.

[8] Paul J Blatz and William L Ko. Application of finite elastic theory to the deformation of rubbery materials. Transactions of the Society of Rheology, 6(1):223-252, 1962.

[9] Ellen M Arruda and Mary C Boyce. A three-dimensional constitutive model for the large stretch behavior of rubber elastic materials. Journal of the Mechanics and Physics of Solids, 41(2):389-412, 1993.

[10] Alan N Gent and AG Thomas. Forms for the stored (strain) energy function for vulcanized rubber. Journal of Polymer Science, 28(118):625-628, 1958.

[11] M Sasso, G Palmieri, G Chiappini, and D Amodio. Characterization of hyperelastic rubber-like materials by biaxial and uniaxial stretching tests based on optical methods. Polymer Testing, 27(8):995-1004, 2008. 
[12] Emilios K Dimitriadis, Ferenc Horkay, Julia Maresca, Bechara Kachar, and Richard S Chadwick. Determination of elastic moduli of thin layers of soft material using the atomic force microscope. Biophysical Journal, 82(5):2798-2810, 2002.

[13] Alvaro Mata, Aaron J Fleischman, and Shuvo Roy. Characterization of polydimethylsiloxane (pdms) properties for biomedical micro/nanosystems. Biomedical Microdevices, 7(4):281-293, 2005.

[14] David C Lin, Emilios K Dimitriadis, and Ferenc Horkay. Elasticity models for the spherical indentation of gels and soft biological tissues. MRS Online Proceedings Library Archive, 1060, 2007.

[15] Alice Chinghsuan Chang and Bernard Haochih Liu. Modified flat-punch model for hyperelastic polymeric and biological materials in nanoindentation. Mechanics of Materials, 118:17-21, 2018.

[16] Li-Heng Cai, Thomas E Kodger, Rodrigo E Guerra, Adrian F Pegoraro, Michael Rubinstein, and David A Weitz. Soft poly (dimethylsiloxane) elastomers from architecture-driven entanglement free design. Advanced Materials, 27(35):5132-5140, 2015.

[17] ID Johnston, DK McCluskey, CKL Tan, and MC Tracey. Mechanical characterization of bulk sylgard 184 for microfluidics and microengineering. Journal of Micromechanics and Microengineering, 24(3):035017, 2014.

[18] Ian M Ward and Dennis W Hadley. An introduction to the mechanical properties of solid polymers. 1993.

[19] F Mujika, N Carbajal, A Arrese, and I Mondragon. Determination of tensile and compressive moduli by flexural tests. Polymer Testing, 25(6):766-771, 2006.

[20] Takanobu Kawamura, Kenji Urayama, and Shinzo Kohjiya. Multiaxial deformations of end-linked poly (dimethylsiloxane) networks 5. Revisit to Mooney-Rivlin approach to strain energy density function. Nihon Reoroji Gakkaishi, 31(4):213-217, 2003.

[21] APS Selvadurai. Deflections of a rubber membrane. Journal of the Mechanics and Physics of Solids, 54(6):1093-1119, 2006. 
[22] Sang-Hee Yoon, Vimalier Reyes-Ortiz, Kwang-Ho Kim, Young Ho Seo, and Mohammad RK Mofrad. Analysis of circular pdms microballoons with ultralarge deflection for mems design. Journal of Microelectromechanical Systems, 19(4):854-864, 2010.

[23] Vlad Cârlescu, Mihai Adrian Rusu, Gheorghe Prisăcaru, and Dumitru N Olaru. experimental characterization and fem simulation on uniaxial tensile and compression of dielectric elastomers.

[24] RM Christensen and WW Feng. Nonlinear analysis of the inflation of an initially flat, circular, elastic disk. Journal of Rheology, 30(1):157-165, 1986.

[25] RS Rivlin. Large elastic deformations of isotropic materials iv. further developments of the general theory. Philosophical Transactions of the Royal Society of London. Series A, Mathematical and Physical Sciences, 241(835):379-397, 1948.

[26] APS Selvadurai and M Shi. Fluid pressure loading of a hyperelastic membrane. International Journal of Non-Linear Mechanics, 47(2):228-239, 2012.

[27] AN Gent. Elastic instabilities in rubber. International Journal of Non-Linear Mechanics, 40(2-3):165-175, 2005. 


\section{Supplementary Information}

Plots for the experimental and analytically obtained curves of best fit for the dimensional and dimensionless data.

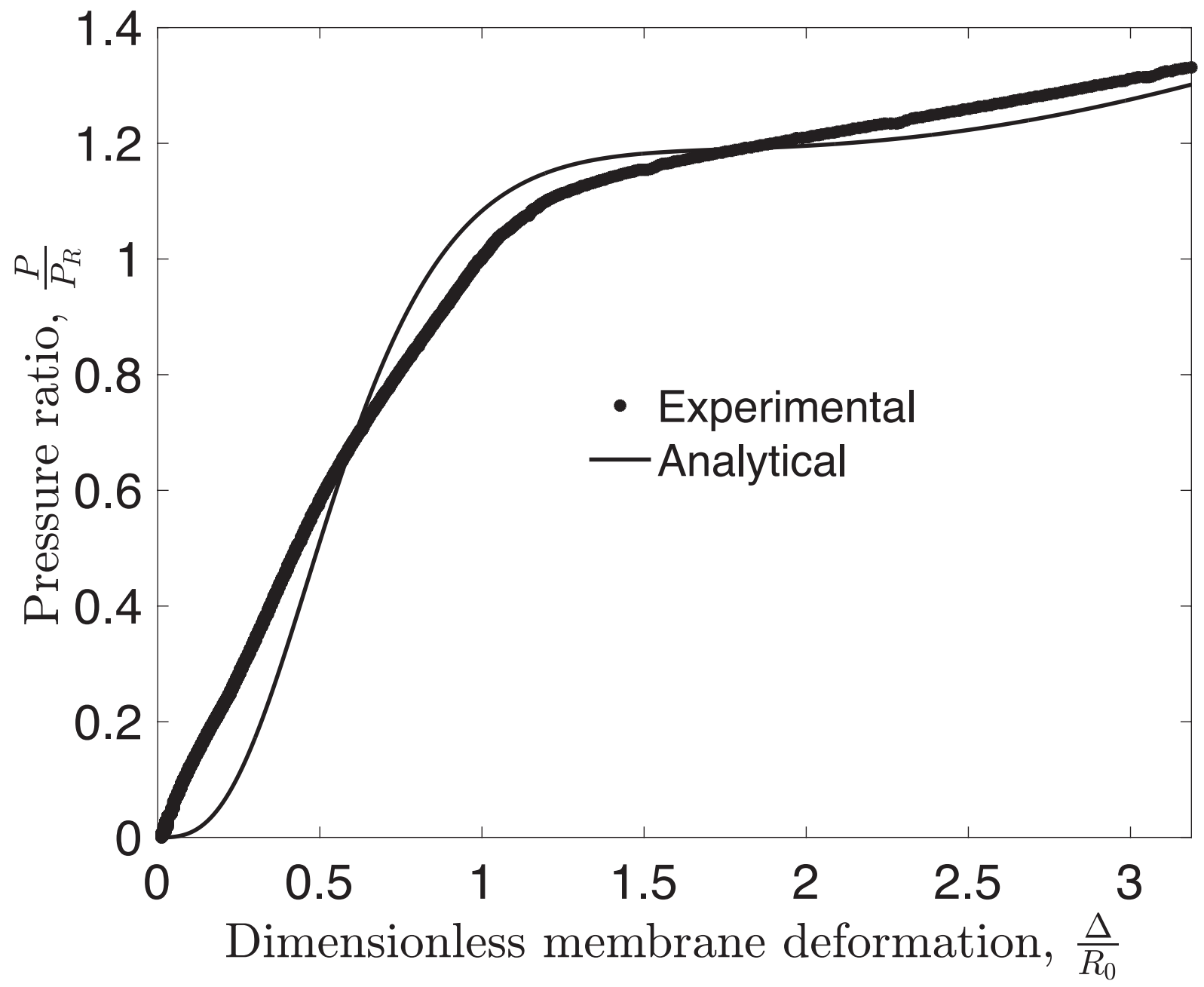

Figure 4: Dimensionless inflation pressure, $P / P_{R}$ plotted with respect to the dimensionless deformation of the membrane, $\Delta / R_{0}$ for Dragon-Skin ${ }^{\mathrm{TM}}$ with the analytically obtained best fit. $\alpha=0.07$, $R^{2}=0.94$ 


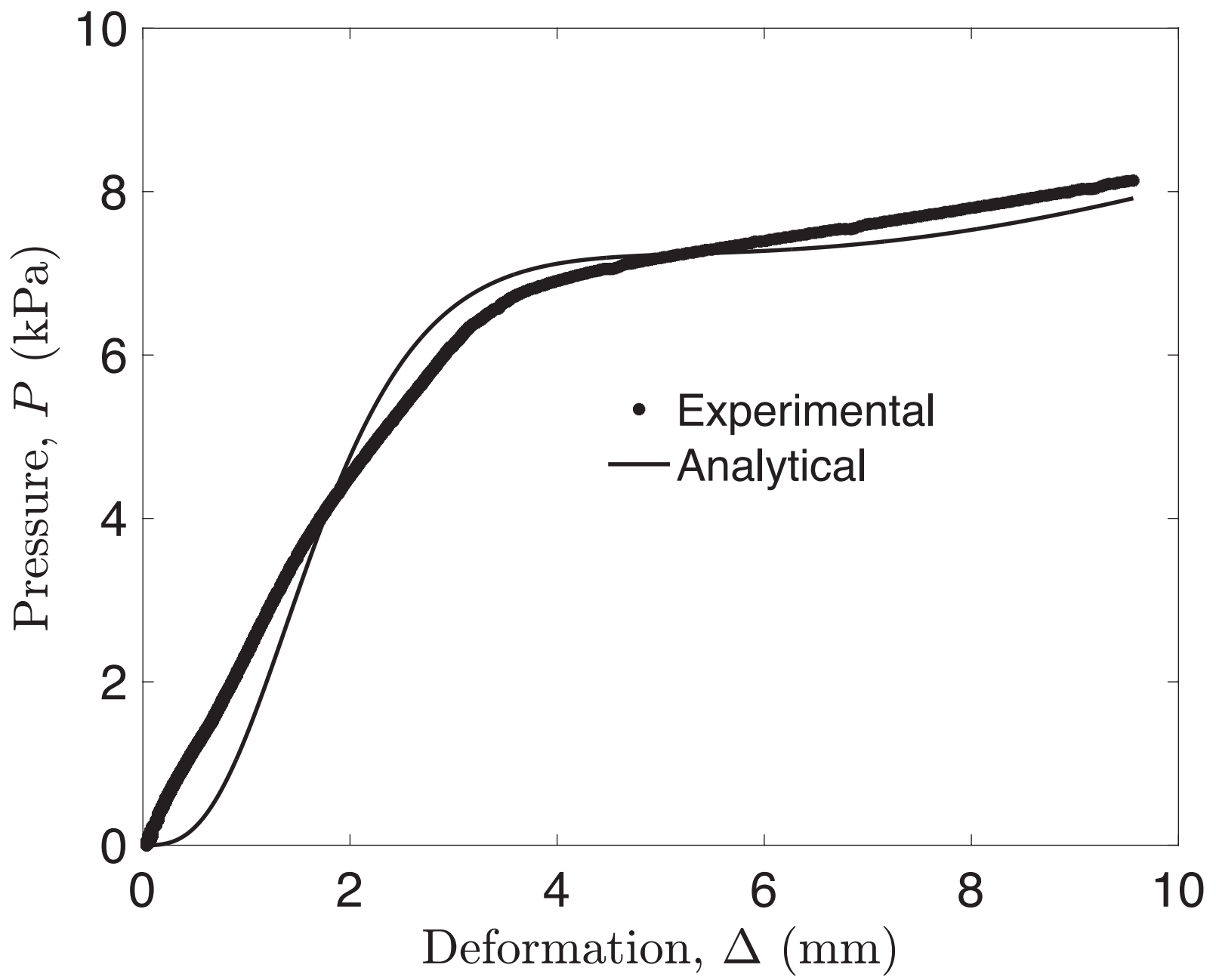

Figure 5: Dimensional inflation pressure, $P$ as a function of the maximum deformation of the membrane, $\Delta$ for Dragon-Skin ${ }^{\mathrm{TM}}$, with the analytically obtained best fit. $R^{2}=0.94$. 


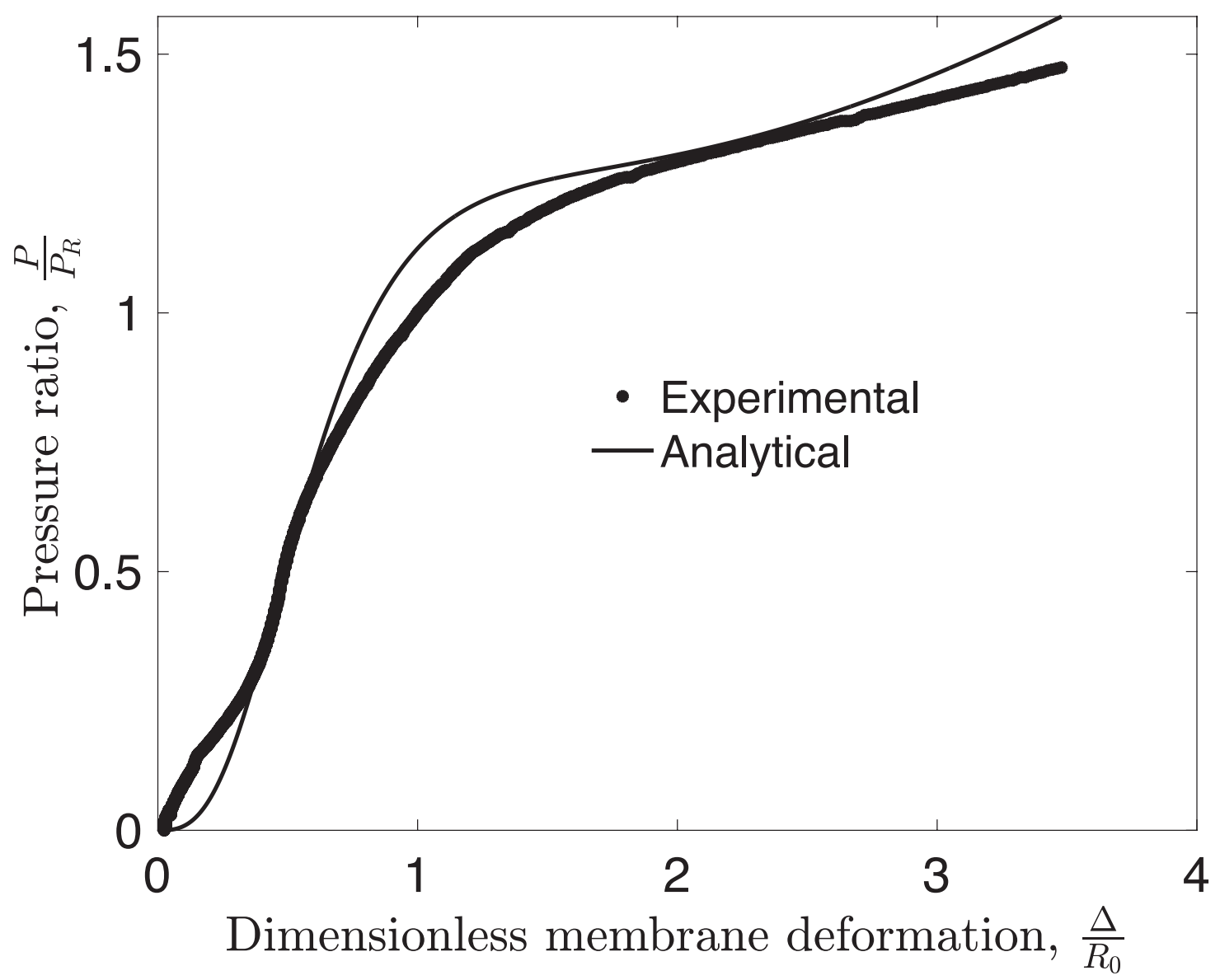

Figure 6: Dimensionless inflation pressure, $P / P_{R}$ plotted with respect to the dimensionless deformation of the membrane, $\Delta / R_{0}$ for Dragon-Skin ${ }^{\mathrm{TM}}+$ Hexane (1:1, by weight) with the analytically obtained best fit. $\alpha=0.08, R^{2}=0.98$. 


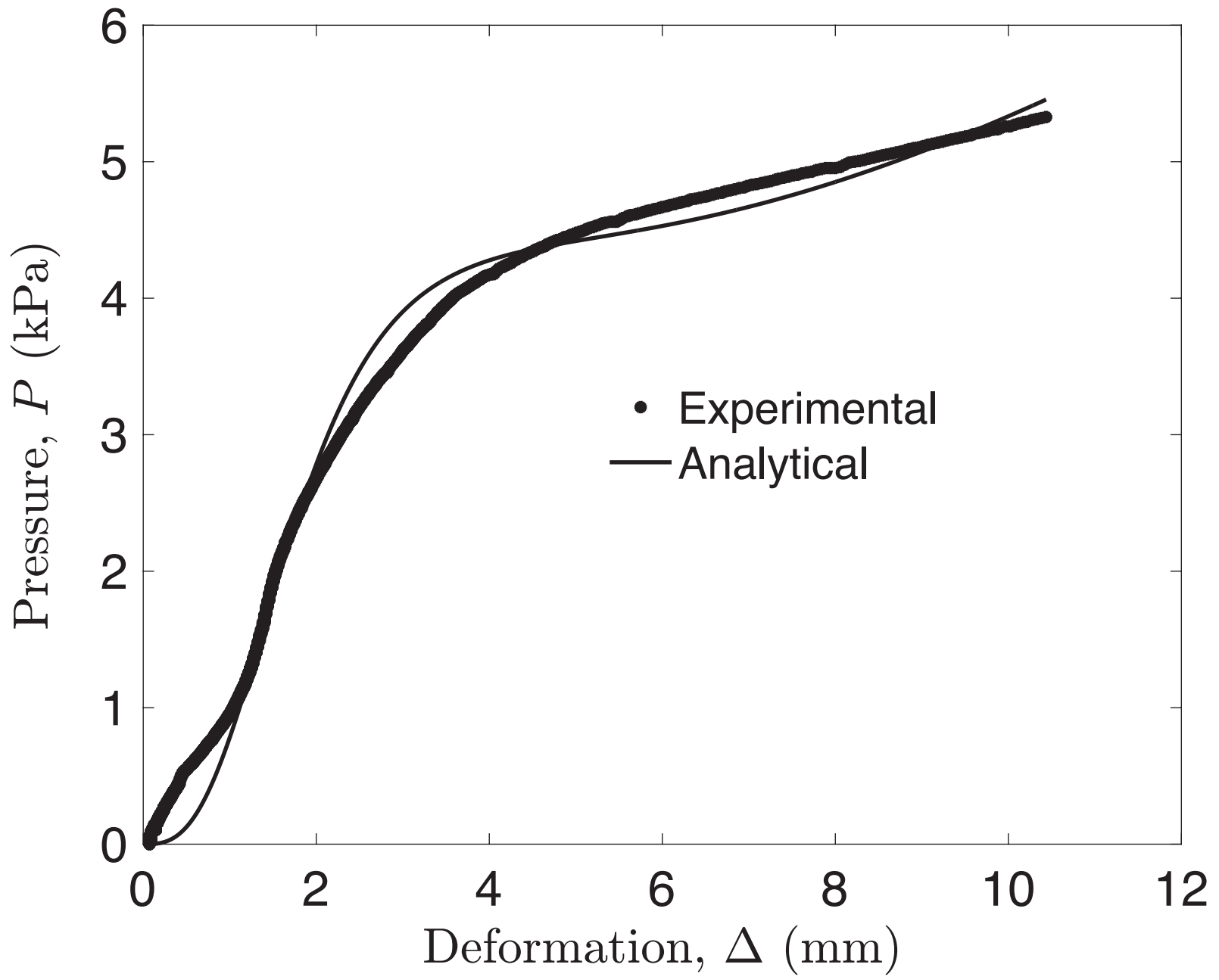

Figure 7: Dimensional inflation pressure, $P$ (y-axis) as a function of the maximum deformation of the membrane, $\Delta$ (x-axis) for Dragon-Skin ${ }^{\mathrm{TM}}+$ Hexane (1:1, by weight) with the analytically obtained best fit. $R^{2}=0.98$. 


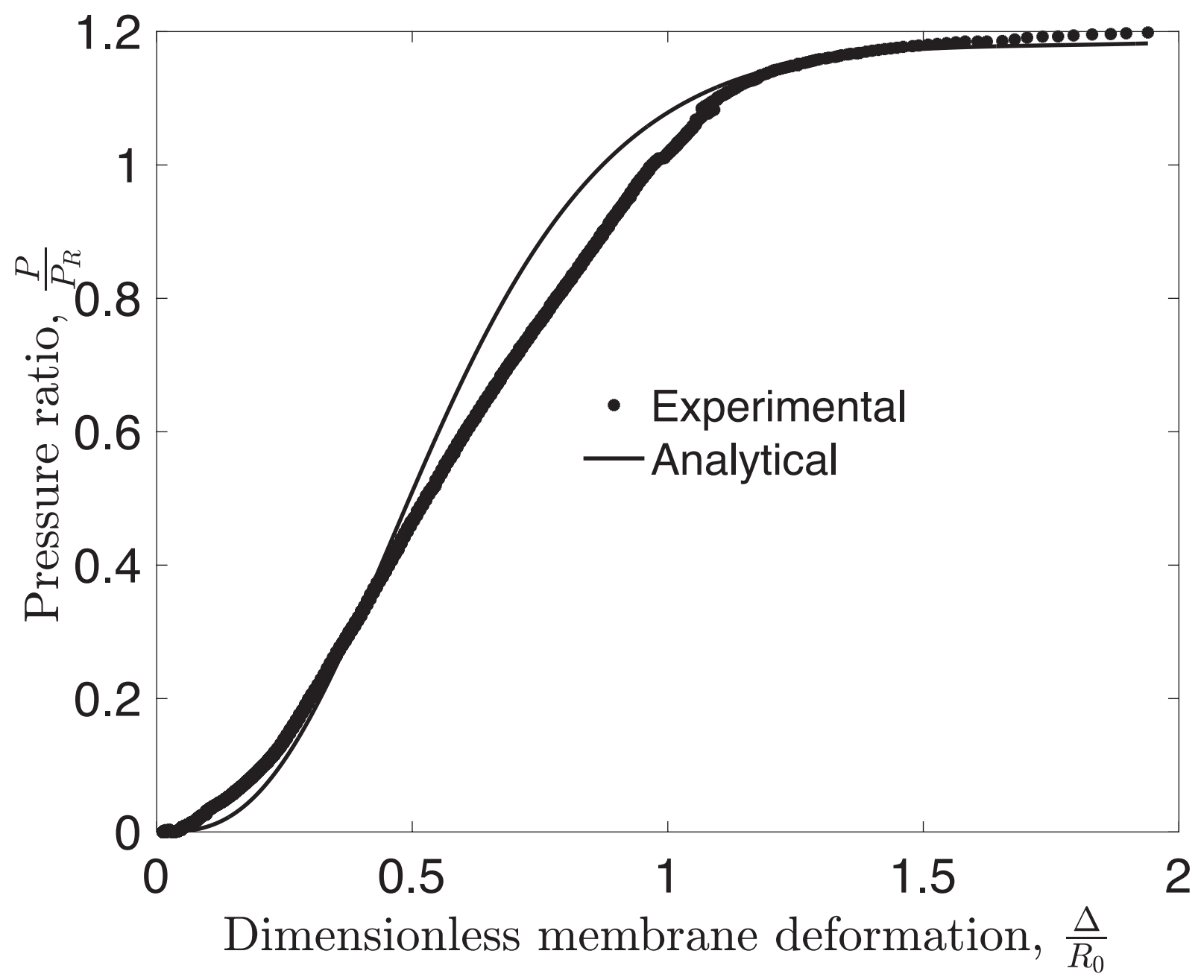

Figure 8: Dimensionless inflation pressure $P / P_{R}$ plotted with respect to the dimensionless deformation of the membrane, $\Delta / R_{0}$ for Sorta-Clear ${ }^{\mathrm{TM}}$ with the analytically obtained best fit. $\alpha=0.06, R^{2}=0.98$. 


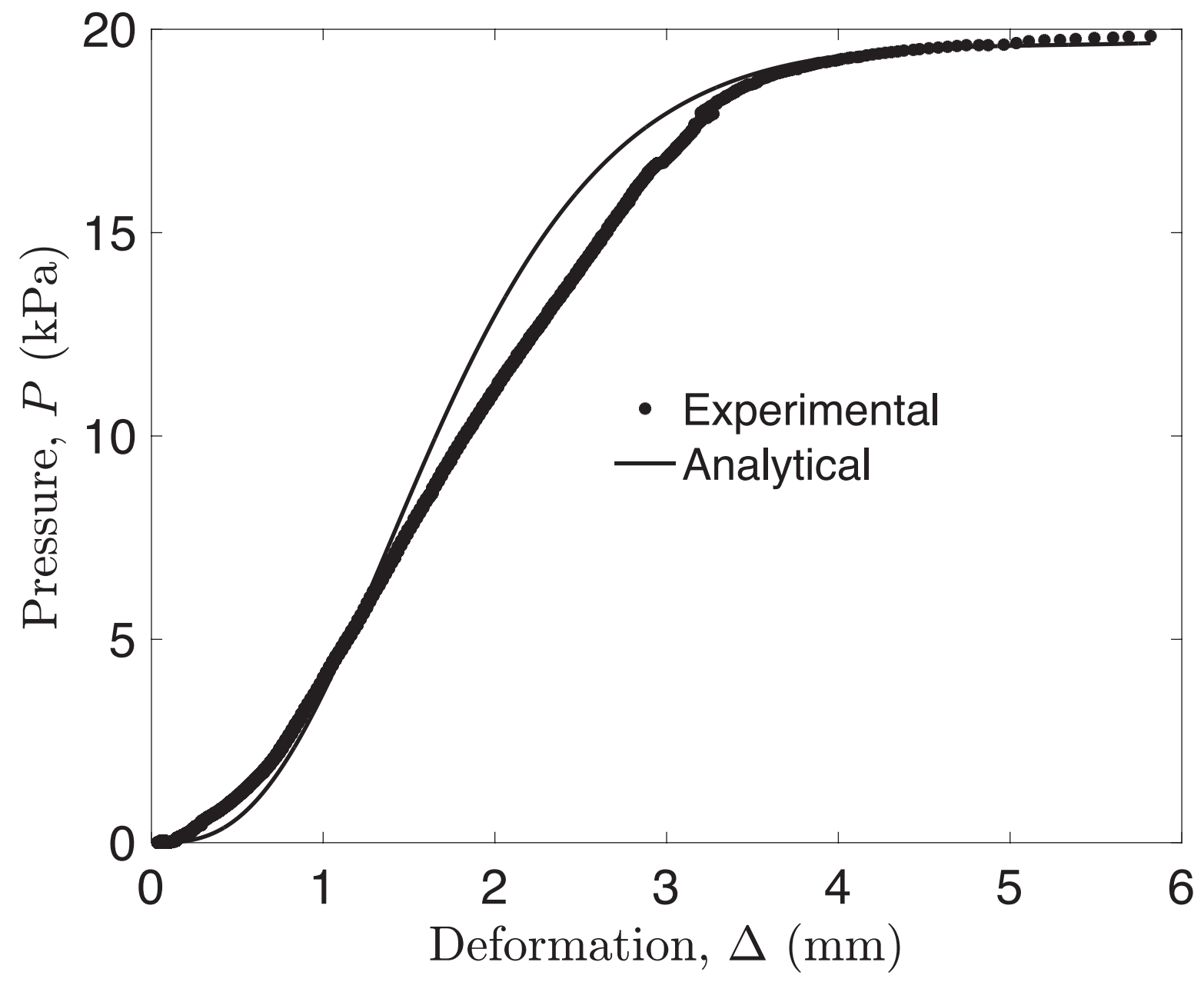

Figure 9: Dimensional inflation pressure, $P$ (y-axis) as a function of the maximum deformation of the membrane, $\Delta$ (x-axis) for Sorta-Clear ${ }^{\mathrm{TM}}$ with the analytically obtained best fit. $R^{2}=0.98$.

$R^{2}$ values for the dimensionless and dimensional data for all five materials tested are provided in Table@, 
Table 3: $\mathrm{R}^{2}$ values obtained through minimization of the least squared error comparing the analytical and experimental data.

\begin{tabular}{|l|c|c|}
\hline \multicolumn{1}{|c|}{ Material } & $\mathrm{R}^{2}$ Dimensionless & $\mathrm{R}^{2}$ Dimensional \\
\hline Dragon-Skin $^{\mathrm{TM}} 10$ & 0.94 & 0.94 \\
Ecoflex $^{\mathrm{TM}}$ & 0.91 & 0.90 \\
Sorta-Clear & 0.98 & 0.98 \\
Sylgard $^{\mathrm{TM}} 184$ (PDMS) & 0.99 & 0.99 \\
Dragon-Skin $^{\mathrm{TM}} 10+$ Hexane(1:1) & 0.98 & 0.98 \\
\hline
\end{tabular}

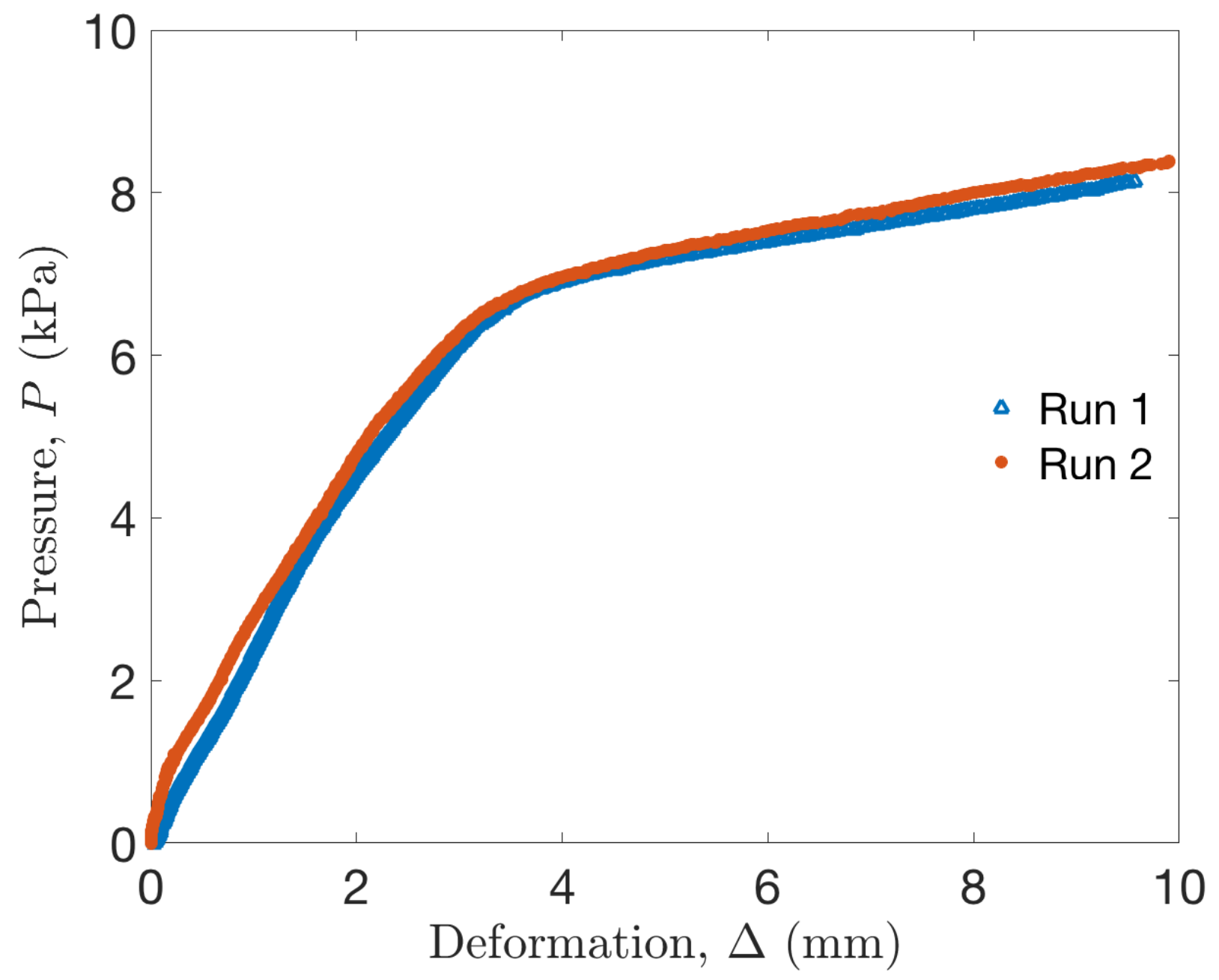

Figure 10: Dimensional inflation pressure, $P$ (y-axis) as a function of the maximum deformation of the membrane, $\Delta$ (x-axis) for Dragon-Skin ${ }^{\mathrm{TM}}$ for separate experiments with the same material. 


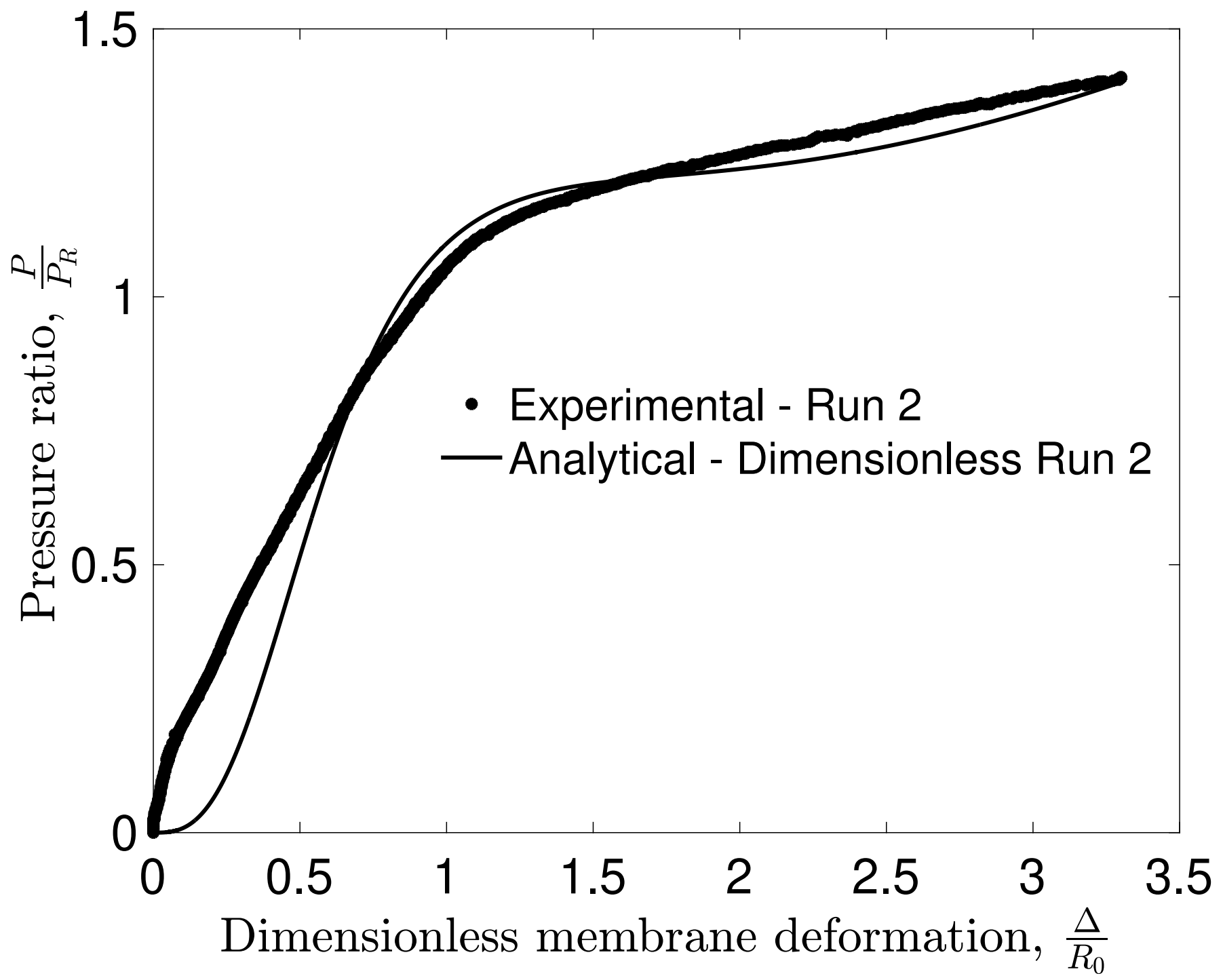

Figure 11: Dimensionless inflation pressure $P / P_{R}$ plotted with respect to the dimensionless deformation of the membrane, $\Delta / R_{0}$ for Dragon-Skin ${ }^{\mathrm{TM}}$ with the analytically obtained best fit. $\alpha=0.072$, $R^{2}=0.95$. (Repeat run.) 


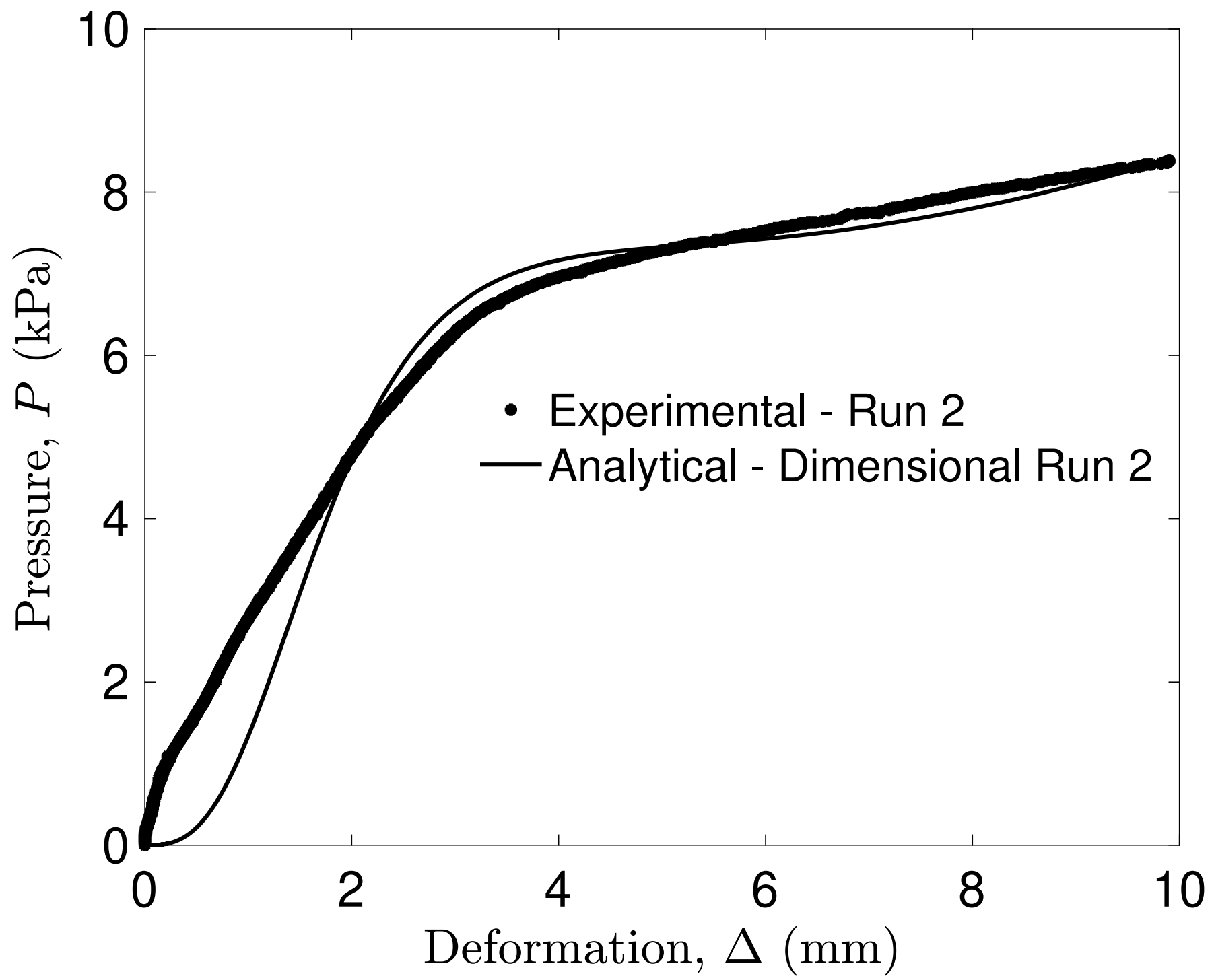

Figure 12: Dimensional inflation pressure, $P$ (y-axis) as a function of the maximum deformation of the membrane, $\Delta$ (x-axis) for Dragon-Skin ${ }^{\mathrm{TM}}$ with the analytically obtained best fit. $R^{2}=0.95 . C_{1}=177.5$ $\mathrm{kPa}, C_{2}=12.78 \mathrm{kPa}$ (Repeat run.) 